\title{
Solving the Spectral Problems by the Modified Method of Successive Approximations
}

\author{
S. M. Yaroshko, S. A. Yaroshko* \\ https://doi.org/10.31174/NT2018-158VI18-04 \\ National University "Lviv Polytechnic", ${ }^{2}$ Ivan Franko National University of Lviv, Lviv, Ukraine \\ ${ }^{*}$ Corresponding author. E-mail: serhiy.yaroshko@lnu.edu.ua
}

Paper received 25.01.18; Accepted for publication 29.01.18.

\begin{abstract}
We describe an effective numerical method of solving the complete spectral problem with a given linear completely continuous operator (or with a polynomial pencil of such operators) acting in a functional normed space. We use the modified method of successive approximations (MMSA) to calculate the eigenvalues (single and multiple), eigenfunctions and associated functions of the operator. We can make a posteriori estimation of the accuracy of the obtained values. The theorem about MMSA convergence and the technique of the accuracy estimation are formulated in this paper. Besides that, some examples of MMSA applications are demonstrated.

Keywords: eigenvalue, eigenfunction, associated function, completely continuous operator, pencil of operators.
\end{abstract}

Introduction. The eigenvalue problems arise in many applied fields of mechanics, electrodynamics, structural analysis, mathematics, quantum mechanics and others. There are many methods, general and special, of solving such problems, but the interest to the question remains at high level. The large number of publications, produced every year, proves this.

We will talk about the modified method of successive approximations (MMSA) [1, 2] designed to calculate the eigenvalues and eigenfunctions of a given linear completely continuous operator. Similarly to the classic power method, MMSA calculates the iterations of some initial function but takes into account not only the two last iterated functions, but all the obtained ones. MMSA acts especially well while the operator has several close characteristic numbers. Recall, that the characteristic number of an operator is reciprocal of the eigenvalue. The method allow us to calculate single and multiple characteristic numbers $\mu_{n}$ with the corresponding eigenfunctions and the associated functions (in case when the geometric multiplicity of a number is less than the algebraic one).

Description of the MMSA. Let $A$ be a linear completely continuous operator acting in a functional normed space $E$. It is known that there exists such entire function $F(\mu)$ that its roots are the characteristic numbers of the operator $A$. It is named the characteristic series of the operator and has the form

$$
F(\mu)=\sum_{j=0}^{\infty} c_{j} \mu^{j} \cdot(1)
$$

Let us consider the spectral problem

$$
u-\mu A u=0,(2)
$$

where $\mu$ is a spectral parameter. Its solutions are the characteristic numbers $\mu_{n}$ and the eigenfunctions $u_{n}(x)$. Let

$$
\left\|u_{n}\right\|=1, n=1,2, \ldots
$$

We consider also the auxiliary problem

$$
u-\mu A u=v_{0} F(\mu),(3)
$$

where $v_{0}$ is some initial function from $E$, the coefficients $c_{j}$ of series (1) are unknown and

$$
v_{0}(x)=\sum_{n=1}^{\infty} b_{n} u_{n}(x), .(4)
$$

Problem (3) has solution at every value of the parameter $\mu$ and becomes a homogeneous spectral problem (2), when $\mu$ becomes equal to a characteristic number $\mu_{n}$. Equation (3) includes $\mu$ polynomially, therefore its solution has a form

$$
u(x)=\sum_{m=0}^{\infty} Z_{m}(x) \mu^{m},(5)
$$

where $Z_{m}(x)=\sum_{j=0}^{m} c_{j} v_{m-j}(x), v_{j}=A v_{j-1}=A^{j} v_{0}$.

Theorem 1. Let all the characteristic numbers $\mu_{n}$ of a linear completely continuous operator $A: E \rightarrow E$ be single, and all the coefficients $b_{n}$ in the decomposition (4) be not equal to zero. If the condition

$$
\lim _{m \rightarrow \infty}\left\|\psi_{m}\right\|=\lim _{m \rightarrow \infty}|| Z_{m} \| /\left|c_{m}\right|=0 \text {, (6) }
$$

is true, then the numbers $c_{j}, j=\overline{0, m}$ in the (5) are the coefficients of an entire function (1), the roots of this function coincide with $\mu_{n}$, and the sum of series (5) at $\mu=\mu_{n}$ is equal to $a_{n} u_{n}$, where $a_{n} \neq 0$. On the contrary, if all $\mu_{n}$ are real positive and growing not slower then $C n^{p}, p>1$ starting from some index $n_{0}$, and condition $\sum_{n=1}^{\infty}\left|b_{n}\right|=K<\infty$ is true, then condition (6) is true.

The proof of the theorem was published in [2]. Theorem 1 gives us the way to construct the computational algorithm. Firstly, we set an initial function $v_{0}$. Next we successively calculate $v_{m}=A v_{m-1}, m=1,2, \ldots$, minimize the function $\psi_{m}$ to obtain the unknown coefficients $c_{j} /\left|c_{m}\right|$ and calculate the roots $\mu_{n}^{(m)}$ of the polynomial $F_{m}(\mu)=\frac{1}{c_{m}^{(m)}} \sum_{j=0}^{m} c_{j}^{(m)} \mu^{j}$ which are the approximate characteristic numbers. Then we calculate the corresponding approximate eigenfunctions as segments of the series (5).

Remark 1. If space $E$ is finite-dimensional then we obtain the exact values of all the characteristic numbers and the eigenvectors for finite amount steps of the algorithm of the method.

Remark 2. If some characteristic number $\mu_{k}$ is $l_{k}$ multiple and has the corresponding eigenfunctions $u_{k, j}(x)$, $j=1, \ldots, l_{k}$, then the function $u_{k}(x)$ in the decomposition (4) has the form $u_{k}(x)=\sum_{j=1}^{l_{k}} a_{j} u_{k, j}(x)$ and the theorem proof remains true. To make sure that the retrieved characteristic numbers are simple we have to solve the problem again with a different initial function. In the new solution the ei- 
genfunction of a multiple characteristic number will differ from the previous one.

Numeric results. The eigenfunctions and eigenvalues of the boundary value problem [3]

$$
-y^{\prime \prime}(x)=\mu y(x), x \in[0 ; 1] ; y(0)=y(1)+y^{\prime}(1)=0,(7)
$$

are $y_{n}(x)=\sin k_{n} x$ and $\mu_{n}=k_{n}^{2}$ respectively, where $k_{n}$ are the roots of the equation $\operatorname{tg} k=-k$. It is easy to construct an integral equation equivalent to (7):

$$
y=A y \equiv \mu \int_{0}^{1} G(x, t) y(t) d t^{\prime} G(x, t)=\left\{\begin{array}{l}
(2-t) x / 2, x \leq t .,(8) \\
t(2-x) / 2, x \geq t
\end{array}\right.
$$

The operator $A$ is completely continuous in the spaces $C[0 ; 1]$ and $L_{2}[0 ; 1]$. The problem (8) was solved by the power method and by MMSA with the same initial function $v_{0}(x) \equiv 1$. The results are shown in the table 1 . It is evident that MMSA provides higher convergence to the first value and calculates several next ones.

Table 1. Eigenvalues of the problem (7) - characteristic numbers of the problem (8)

\begin{tabular}{|c|r|c|c|c|c|}
\hline \multirow{2}{*}{ Iteration, $n$} & Power method, & \multicolumn{4}{|c|}{ MMSA } \\
\cline { 3 - 6 } & $\mu_{1}^{(n)}$ & $\mu_{1}^{(n)}$ & $\mu_{2}^{(n)}$ & $\mu_{3}^{(n)}$ & $\mu_{4}^{(n)}$ \\
\hline 1 & 4,8 & 4,166666667 & & & \\
2 & 4,1667 & 4,116062477 & 31,43418628 & & \\
3 & 4,12270 & 4,115858528 & 24,37850638 & 87,85765293 & \\
4 & 4,116932 & 4,115858366 & 24,14142238 & 65,26022330 & 235,4016604 \\
5 & 4,1160399 & 4,115858366 & 24,13935078 & 63,73463220 & 139,3090487 \\
6 & 4,11588829 & 4,115858366 & 24,13934205 & 63,66050780 & 124,5039154 \\
\hline Right values & 4,115858366 & 4,115858366 & 24,13934203 & 63,65910653 & 122,8891618 \\
\hline
\end{tabular}

Note, that the eigenfunctions $y_{n}$ do not establish a basis of $C[0 ; 1]$ but MMSA works successfully.

Let us consider the spectral problem with the simple-layer potential

$$
\mu \int_{C} \ln \rho\left(s, s^{\prime}\right) u\left(s^{\prime}\right) d s^{\prime}=u(s),(9)
$$

where $C$ is a circle of the given radius $R$ (let $R$ be equal to 1); $s, s^{\prime}$ - points on the circle; $\rho\left(s, s^{\prime}\right)$ - distance between them. In the polar coordinate system $(r, \varphi)$ the equation (9) becomes

$$
\mu \int_{-\pi}^{\pi} \ln \sqrt{2\left(1-\cos \left(\varphi-\varphi^{\prime}\right)\right)} u\left(\varphi^{\prime}\right) d \varphi^{\prime}=u(\varphi),
$$

It is easy to verify that the characteristic numbers of (10) are $\mu_{n}=-n / \pi, n=1,2, \ldots$ with the corresponding eigenfunctions $u_{n, 1}=\cos n \varphi, u_{n, 2}=\sin n \varphi$, i.e. every $\mu_{n}$ is twomultiple. We solved (10) in $L_{2}[-\pi ; \pi]$ by MMSA with different initial functions $v_{0}$ (even, odd and general) and obtained the $\mu_{n}$ as simple numbers but with the different corresponding eigenfunctions. The results are shown in table 2 .
The approximate eigenfunctions $u_{1}, u_{2}, u_{3}, u_{4}$ have the accuracies $10^{-9}, 10^{-7}, 10^{-4}, 10^{-2}$ respectively.

The initial function $v_{0}=\varphi \sin \varphi$ is even, so its decomposition of form (4) contains only $u_{n, 1}$ eigenfunctions and the corresponding coefficients have the form $b_{n}=\int_{-\pi}^{\pi} \varphi \sin \varphi \cos n \varphi d \varphi=-\pi / 2, n=1 ; 2 \pi \frac{(-1)^{n+1}}{n^{2}-1}, n \geq 2$.

It is easy to make sure that theorem 1 restriction on $b_{n}$ is satisfied: $\quad \sum_{n=1}^{\infty}\left|b_{n}\right|=\frac{\pi}{2}+2 \pi \sum_{n=2}^{\infty} \frac{1}{n^{2}-1}=2 \pi$. Conversely, the initial function $v_{0}=\varphi \cos \varphi$ is odd, its coefficients $b_{n}=\int_{-\pi}^{\pi} \varphi \cos \varphi \sin n \varphi d \varphi=-\pi / 2, n=1 ; 2 \pi \frac{(-1)^{n} n}{n^{2}-1}, n \geq 2$, and the series $\sum_{n=1}^{\infty}\left|b_{n}\right|$ is divergent. This example demonstrates that the restriction on $b_{n}$ is not obligatory in the applications.

Table 2. Characteristic numbers and eigenfunctions of the problem (10)

\begin{tabular}{|l|l|l|l|l|c|}
\hline \multirow{2}{*}{$n$} & \multirow{2}{*}{ Exact $\mu_{n}$} & $\begin{array}{c}\text { Approximate } \\
\mu_{n}\end{array}$ & \multicolumn{3}{|c|}{ Approximate $u_{n}$ obtained with different $v_{0}(\varphi)$} \\
\cline { 4 - 6 } & & $v_{0}=\varphi \sin \varphi$ & $v_{0}=\varphi \cos \varphi$ & $v_{0}=\exp \varphi \sin \varphi$ \\
\hline 1 & -0.3183098862 & -0.3183098862 & $u_{1}=\cos \varphi$ & $u_{1}=\sin \varphi$ & $u_{1}=a \cos \varphi+b \sin \varphi$ \\
\hline 2 & -0.6366197724 & -0.63661978 & $u_{2}=\cos 2 \varphi$ & $u_{2}=\sin 2 \varphi$ & $u_{2}=a \cos 2 \varphi+b \sin 2 \varphi$ \\
\hline 3 & -0.9549296586 & -0.9550 & $u_{3} \approx \cos 3 \varphi$ & $u_{3} \approx \sin 3 \varphi$ & \\
\hline 4 & -2.683239545 & -2.69 & & & $a \approx-0.4479 ; b \approx 0.8941$ \\
\hline
\end{tabular}

Both operators in the problems above were self-adjoint. Let us consider another problem with a not self-adjoint operator:

$$
y^{\prime \prime}(x)=\mu y^{\prime}(x), \quad x \in[0 ; 1], \quad y(0)=y(1)=0,(11)
$$

It is known [3] that the eigenvalues of (11) are complex conjugated numbers $\mu_{k}=2 k \pi i, k= \pm 1, \pm 2, \ldots$ We transformed the problem (11) to the integral form using the Green's function $G(x, t)=\left\{\begin{array}{cc}-x, & x \leq t \\ 1-x, & x \geq t\end{array}\right.$ and solved it by MMSA. The kernel of the integral operator is simple enough so we can calculate the functions $v_{j}$ and the coefficients $c_{j}$ analytical- ly (with the initial function $v_{0}(x)=x$ ) and diminish numerical errors. We performed thirty-seven steps of the method and obtained twenty-four approximate characteristic numbers. The first pair of them have twenty-four right decimal digits and the last pair - only two digits.

It turned out that the approximate solutions converge to the exact values as a geometric progression with a small common ratio $q$, while the steps of MMSA are executed analytically. We saw in different problems that the value of $q$ varies from 0.001 to 0.1 . 
Associated function. Sometimes the algebraic multiplicity of a characteristic number is greater than the amount of the corresponding eigenfunctions. In this case, the associated functions correspond to the number as well. For instance, let $\mu_{k}$ be a two-multiple number with a single eigenfunction $u_{k}$. Then the associated function $w_{k}$ corresponds to $\mu_{k}$. This function is a solution of the equation $A w_{k}-\mu_{k}^{-1} w_{k}=u_{k}$.

It turns out [2] that MMSA can calculate such numbers and functions if the initial function has the form $v_{0}(x)=b_{k}^{(1)} w_{k}(x)+\sum_{n=1}^{\infty} b_{n} u_{n}(x)$ and the algorithm is complemented with formulas for the associated function: $\boldsymbol{w}_{k}^{(m)}=\sum_{j=1}^{m-1} T_{j}^{(m)} j \boldsymbol{\mu}_{k}^{(m) j-1}, T_{j}^{(m)}=\sum_{i=j+1}^{m} c_{i} v_{m+j-i}$. We verified it

Error estimation. It is shown in [4] that at the $m$-th step of the algorithm we need to solve the system

$\mathbf{\Lambda} \overrightarrow{\boldsymbol{c}}^{(m)}=-\vec{e},(12)$

where $\Lambda=\left\{\Lambda_{i j}\right\} ; \Lambda_{i j}=\mu_{i}^{-j} ; \vec{e}=(1, \ldots, 1) ; c_{j}^{(m)}$ is the $m$ th approximation to the unknown coefficients $c_{j}$. Some elements $\Lambda_{i j}$ vanishes during the calculations due to the finite computer memory. We mark $\boldsymbol{\Lambda}_{\delta}$ remaining in the memory part of the matrix $\Lambda$. Then the linear part of the method error has form

$$
\vec{\varepsilon}=R(\mu) \equiv \mathbf{P}^{-1}\left(\vec{c}^{(m)}+\mathbf{\Lambda}_{\delta}^{-1} \vec{e}\right),
$$

where $\mathbf{P}-m \times m$ matrix, $i$-th column of it consists of the coefficients of the polynomial $\prod_{j=1, j \neq i}^{m}\left(\mu-\mu_{j}\right)$.

Whereas the exact numbers $\mu_{j}$ are unknown, we use the obtained approximate numbers $\mu_{\delta j}$ to estimate the method error in the way: 1) calculate $\varepsilon_{\delta}=R\left(\mu_{\delta}\right)$; 2) calculate $\left.\varepsilon^{-}=R\left(\mu_{\delta}-\varepsilon_{\delta}\right), \varepsilon^{+}=R\left(\mu_{\delta}+\varepsilon_{\delta}\right) ; 3\right)$ write the estimation as intervals $\delta_{j} \in\left[\varepsilon_{j}^{-} / \mu_{\delta j}, \varepsilon_{j}^{+} / \mu_{\delta j}\right], \quad j=1, \ldots, m$. by solving several model problems.

The integral operator $A u \equiv \sqrt{k / 2 \pi} \int_{-1}^{1} \cos (k x t) u(t) d t$ defines the Fourier cosine transform of finite functions.It is known, that the absolute values of characteristic numbers of this operator gather near 1 when the parameter $k$ becomes larger. The first five characteristic numbers are tabulated for $k=1, \ldots, 8$. We calculated them by MMSA and estimated its accuracy according to the described technique. All the obtained number and estimations of them coincided with the known values.

An interesting result was obtained for $k=16$ : $\mu_{\delta 1}=1.0000000, \quad \mu_{\delta 2}=-1.0000000, \quad \mu_{\delta 3}=1.0000011$, $\mu_{\delta 4}=-1.0003014, \quad \mu_{\delta 5}=1.0265150, \quad \mu_{\delta 6}=-1.6333306$, $\mu_{\delta 7}=8.0776840, \quad \mu_{\delta 8}=-79.6164, \quad \mu_{\delta 9}=1118.5$; $\delta_{1}=\ldots=\delta_{6}=0, \quad \delta_{7} \in[0.08 \% ; 0.09 \%], \quad \delta_{8} \in[0.12 \% ; 0.13 \%]$, $\delta_{9} \in[6.42 \% ; 6.44 \%]$.

Polynomial pencil of operators. It is possible to solve a spectral problem with a polynomial pencil of completely continuous operators by MMSA. According to [5] we can replace the original problem by the linearized one and solve it by the method. For instance, let consider a problem $B_{3} \mu^{3}+B_{2} \mu^{2}+B_{1} \mu+I=0$, where the operators $B_{i}$ act in a normed space $E$ and $I$ is a unite operator. The equivalent problem has form (2) assuming that the operator $A$ acts in the space $E \oplus E \oplus E$ and has form $A=\left(\begin{array}{ccc}0 & I & 0 \\ 0 & 0 & I \\ -B_{3} & -B_{2} & -B_{1}\end{array}\right)$. This approach was tested on a wide range of problems with the pencils of matrices and operators. The highest power of matrix pencil was eight and the power of operator pencil three. We obtained right solutions in all the cases.

Conclusions. The modified method of successive approximations is used to solve spectral problems with matrix or integral operators and with polynomial pencils of such operators. The method calculates several eigenvalues and eigenfunctions of the problem. The domain of application of MMSA is wider in practice then in theory, as described in the grounding theorem 1. Most effectiveness of the method is reached when several primary eigenvalues are close. Accuracy of the approximate solution can be estimated after finishing the calculations. The estimation technique takes into account the peculiarities of MMSA.

\section{REFERENCES}

1. Voitovich M.M., Yaroshko S.M., Yaroshko S.A. Posteriori Estimation of Calculation of Characteristic Numbers in Modified Successive Approximation Method. Mathematical methods and physical-mechanical fields, 2000, Vol. 43, No. 1. P. 59-67. (in Ukrainian)

2. Yaroshko Svitlana About Grounding of The Modified Method of Successive Approximations for Calculating Completely Continuous Operator Characteristic Numbers. Visnyk Lviv Univ Ser. Applied Mathematics and Computer Science. 2001. No. 4. P. 7179. (in Ukrainian)

3. Collatz, Lothar Eigenwertaufgaben mit technischen Anwendungen - Akadem. Verl.-Ges. Geest \& Portig, 1963.

4. Yaroshko Svitlana, Yaroshko Sergiy Methodic of Posteriori Estimation of Accuracy of the Modified Method of Successive Approximations Visnyk Lviv Univ Ser. Applied Mathematics and Computer Science. 2005. No. 10. P. 49-58. (in Ukrainian)

5. Yaroshko Svitlana, Yaroshko Sergiy Expansion of the Modified Method of Successive Approximations on Spectrum Problems with Polynomial Operator Pencils. Visnyk Lviv Univ Ser. Applied Mathematics and Computer Science. 2008. No. 14. P. 170176. (in Ukrainian)

\section{Решение задач на собственные значения модифицированным методом последовательных приближений}

\section{С. М. Ярошко, С. А. Ярошко}

Аннотация. Описан эффективный численный метод решения полной спектральной задачи для заданного линейного вполне непрерывного оператора (или полиномиального пучка таких операторов), действующего в нормированном функциональном пространстве. Модифицированный метод последовательных приближений используют для вычисления собственных значений (простых и кратных), собственных и присоединенных функций оператора. Точность вычисленных приближенных значений можно оценить апостериори. В статье сформулирована теорема, обосновывающая сходимость метода, описана методика апостериорной оценки точности. Приведены примеры применения метода.

Ключевые слова: собственное значение, собственная функиия, присоединенная функиия, вполне непрерывный оператор, операторный пучок. 